Einführung in das Schwerpunktthema

\title{
Kommunikation konstituiert nachhaltige Regionalentwicklung
}

$\mathrm{N}$

Von Reimar Molitor und Guido Nischwitz Begriff seit fast einer Dekade - Grund genug zum runden Geburtstag ein Zwischenfazit zu ziehen. Die Zusammenführung der inhaltlichen Dimensionen des Entwicklungsleitbildes Nachhaltigkeit und des operativen Geschäfts regionaler Entwicklung hat in den vergangenen zehn Jahren quer durch Europa viele Gesichter hervorgebracht, teilweise auch existierende Gesichter regionaler Entwicklung verändert. Versuche des Vergleichs und der Systematisierung haben dabei Folgendes deutlich gemacht: Die Übersetzung bzw. Umsetzung der regulativen Idee der Nachhaltigkeit in räumlichen Entwicklungen produziert eine Fülle von Ansätzen, Konzepten und Projekten auf regionaler Ebene (1). Der kleinste gemeinsame Nenner dieser theoretischen und praktischen Übersetzungsversuche ist das Bekenntnis zum Leitbild selbst - die inhaltliche und organisatorische Heterogenität der Ansätze, Konzepte und Projekte deutet jedoch darauf hin, dass die Interpretationen des Leitbildes stark variieren.

\section{Region + Nachhaltigkeit = nach- haltige Regionalentwicklung?}

Es herrscht immer noch Unklarheit darüber, was der Begriff nachhaltige Regionalentwicklung im Kern enthält. Diese Unklarheit hat nachvollziehbare Ursprünge: Zum einen gibt es schon seit Mitte der 70er Jahre einen kontinuierlichen, formalen Bedeutungszuwachs der Raumebene Region im Europa der Regionen. Dieser hat einen schleppend vorangehenden Wandel jener europäischen und nationalen Entwicklungsansätze und -politiken mit sich gebracht, die in der Literatur mit der Steuerungsrichtung , von oben " hinterlegt sind. Zum anderen vollzieht sich seit Ende der 70er Jahre eine verstärkte Emanzipation der Raumebene Region in Europa von unten her. Im Rahmen regionaler und teilweise informeller Selbstorganisationsmuster reagieren Akteure verschiedenster Couleur auf zentralisierte und ihrer Meinung nach unadäquate Steuerung von oben und gewinnen Gestaltungsmacht bei der Entwick- lung ihrer Region zurück. Dabei ist meist die Identifikation ökonomischer, ökologischer und/ oder sozialer Schieflagen innerhalb einer Region Ausgangspunkt dieser Bottom-up-Ansätze. Beide zuvor geschilderten Bewegungen sind seit Rio 1992 mit dem Terminus nachhaltige Entwicklung konfrontiert. Während die pure Begrifflichkeit sehr schnell und pragmatisch Eingang in das Vokabular der laufenden Prozesse und Projekte fand, ist der Stand der inhaltlichen und konzeptionellen Adaption der Anforderungen einer nachhaltigen Regionalentwicklung weiterhin fraglich. Eines lässt sich dennoch mit Sicherheit konstatieren: Es tut sich eine ganze Menge unter diesem begrifflichen Dach: von den programmatischen Aussagen der European Spatial Development Perspective, der prozess-orientierten Ausrichtung der europäischen Instrumente LEADER+ und INTERREG, über die aktivierenden Wettbewerbe des Bundes (siehe unten), den landesspezifischen Aktivitäten zur Entwicklung von Regionen (vgl. zum Beispiel den Prozess Zukunftsfähiges ScbleswigHolstein oder das Förderinstrument REGIONALE in Nordrhein-Westfalen) bis hin zu den vielen best practices vor Ort - Region und Nachbaltigkeit passen und gehören offensichtlich zusammen. Aber warum? Auf der Suche nach Antworten auf diese Frage treten zwei Sachverhalte ins Bild:

- Sowohl das Leitbild der Nachhaltigkeit als auch das operative Geschäft regionaler Entwicklung thematisieren zentral die Gestaltung der Zukunft.

- Die nachhaltigen Integrationsansprüche der Entwicklungsdimensionen Ökonomie, Ökologie und soziale Gerechtigkeit finden auf der Raumebene Region die Möglichkeit einer operationalisierten, koordinierten Zusammenführung.

Im Laufe der vergangenen zehn Jahre wurden diesen Basis-Sachverhalten Ziele an die Seite gestellt, die dem scheinbar beliebig fuillbaren Begriff nachhaltige Regionalentwicklung den Status eines verfolgbaren Konzepts räumlicher Entwicklung verliehen. Diese Ziele einer nachhaltigen Regionalentwicklung wurden sowohl theoretisch aus den Rio-Dokumenten abgeleitet als auch sukzessive um inhaltliche Dimensionen eigenständiger Regionalentwicklung ergänzt (vgl. den Kasten).
Ziele einer nachhaltigen Regionalentwicklung

- Nutzung regionaler Ressourcen (Importsubstitution)

- Aufbau regionaler Wirtschaftskreisläufe

- Reduzierung der großräumigen Arbeitsteilung

- Räumliche Bindung der Wertschöpfung

- Abbau ökonomischer Disparitäten innerhalb und zwischen Regionen

- Verringerung der Abhängigkeit von Subventionen

- Reduzierung der Freiflächeninanspruchnahme für

Siedlungszwecke

- Reduzierung des städtischen und regionalen Stoffund Energieverbrauchs

- Inter- und intraregionale Gerechtigkeit

- Förderung sozialräumlicher Mischung

- Förderung der regionalen Eigenständigkeit

- Erhöhung der lokalen und regionalen Handlungs- und Problemlösungskompetenzen

- Förderung dezentraler, selbstverwalteter Planungsund Entscheidungsstrukturen (Subsidiaritätsprinzip)

Quelle: erweitert nach Kühn, M./ Moss, T. (Hrsg.): Planungskultur und Nachhaltigkeit. Berlin 1998

Die Ausgestaltung dieser Ziele vor Ort wurde dabei aus zwei Richtungen befördert:

1. Von unten durch die zahlreichen regionalen Prozesse und Projekte, die in fast ebenso zahlreichen good und best practices-Sammlungen gelistet sind;

2. durch die Promotion der oben genannten Ziele in Programmen von oben, wobei sich das oben insbesondere auf die europäischen, nationalen und länderspezifischen Förderprogramme und -instrumente bezieht.

Besondere Beachtung verdient in diesem $\mathrm{Zu}-$ sammenhang die zunehmende Anzahl an Wettbewerben zur Initiierung und Unterstïtzung regionaler Entwicklung. Von LEADER und INTERREG über Regionen der Zukunft, InnoRegio und REGIONEN Aktiv wurden und werden Regionen gesucht und gefunden, die sich aufmachen, nachhaltige Entwicklungspotenziale zu thematisieren und für sich zu erschließen.

\section{Neue Kommunikation - neve Regionen?}

Eine Gemeinsamkeit dieser vielen Programme, Prozesse und Projekte unter dem Dach nachhaltige Regionalentwicklung ist die (teilweise explizit eingeforderte) Neukonstellation der am Entwicklungsprozess beteiligten Akteure. Die damit verbundenen Kommunikationsprozesse gewinnen immer mehr an Bedeutung (vgl. auch folgenden Kasten). 
KOMREG - Kommunikation für eine nachhaltige Entwicklung in der Region

Im Mittelpunkt des von der Deutschen Bundesstiftung Umwelt (DBU) finanzierten Tagungs- und Forschungsprojekts $(2000 / 2001)$ stand die Identifizierung von Ansätzen, wie Kommunikations- und Entscheidungsprozesse im Sinne des Zielkatalogs der Nachhaltigkeit optimiert und bestehende Kommunikationsbarrieren bzw. Handlungsblockaden auf regionaler Ebene abgebaut werden können. Von zentraler Bedeutung war die Bearbeitung der drei Kernelemente einer nachhaltigen Regionalentwicklung: Ziele, Akteure und Instrumente (3).

Die Grundfragen

- Wer darf (nachhaltige) regionale Zukünfte thematisieren, strategisch formulieren und darüber entscheiden?

- Wie läuft das Verfahren der Konsensfindung bzw. der Festlegung regionaler Entwicklungsziele ab?

- Welche (rechtlichen) Verbindlichkeiten und finanziellen Möglichkeiten haben neue Akteurskonstellationen?

befinden sich dabei bis heute in einem stetigen (regionalen) ,trial-and-error"-Prozess (2).

Eine systematische Aufarbeitung der identifizierten Ziele, der eingesetzten Instrumente, der beteiligten Akteure usw. soll hier nicht geleistet werden (3). Nur eines muss an dieser Stelle hervorgehoben werden: Das Verständnis des Begriffs Region wird im Prozess der Etablierung einer nachhaltigen Regionalentwicklung modifiziert bzw. neu bestimmt: In Bezug auf die verschiedenen thematischen Dimensionen nachhaltiger Regionalentwicklung lässt sich festhalten, dass jeweils unterschiedliche Themen regionaler Entwicklung (z.B. Ver- und Entsorgung, Verkehr, ...) auch themenspezifische Regionszuschnitte verlangen. Gemäß der Integration der inhaltlichen Dimensionen Ökologie, Ökonomie und soziale Gerechtigkeit (Zieldreieck der Nachhaltigkeit) für eine nachhaltige Entwicklung, müssen dabei sowohl

\section{Flusseinzugsgebiete: Rückbindung der Wirtschaft an die Natur}

ist das Thema des Informationsdienst

\section{Ökologisches Wirtschaften 2/02}

Wenn Sie potenzielle Beiträge haben, wenden Sie sich bitte an die Redaktion.
- stoffliche Raumdimensionen (Stoff- und Energiekreisläufe),

- funktionale Raumdimensionen (Wirtschaftskreisläufe, Austauschbeziehungen) als auch

- natur- und kulturräumliche Dimensionen (Topographie, Identität)

in einem neuen, flexiblen Verständnis von Region zusammengeführt werden.

Ausdruck finden diese neuen thematischen Regionen derzeit schon in regionalen Raumkategorien, die das administrative Raumkonstrukt Region in seiner territorialen Dimension modifizieren. Für die Vielschichtigkeit dieser Modifikation stehen folgende Auftritte stellvertretend: Wirtschaftsregion, Zukunftsregion, Programmregion, Kulturregion, Kooperationsraum, Modellregion, Technologie-Region, Metropolregion, Großraum, Teilraum, Städtekranz, Städtequartett, Städtedreieck, Werkstattregion, ...

Damit unmittelbar verbunden ist die eindeutig beobachtbare Neukonstellation von regionalen Akteuren. Gemäß der themenspezifischen Raumzuschnitte ist sowohl auf europäischer als auch auf nationaler Ebene eine themenspezifische Vernetzung von Akteuren zu beobachten, welche Kompetenz- und Zuständigkeitsfragen aufwirft. Nicht selten etablieren und institutionalisieren sich neue Kommunikations-, und damit Macht- und Entscheidungsstrukturen, die teilweise jenseits der etablierten Planungsträger (europäisch, nationalstaatlich sowie regional) arbeiten. Die dauerhafte Belastbarkeit dieser neuen Strukturen muss sich jedoch erst noch erweisen.

\section{- Die Beiträge im Überblick}

Im Rahmen des vorliegenden Schwerpunkts wird der vielschichtige Zusammenhang zwischen Kommunikation, Nachhaltigkeit und Region aus unterschiedlichen Blickwinkeln heraus erörtert. Der erste Beitrag vonJoe Ravetz aus Manchester beschäftigt sich mit dem Bedarf an neuen, kommunikationsorientierten Instrumentensets für eine nachhaltige Regionalentwicklung. Vor dem Hintergrund vielfältiger Erfahrungen und Erkenntnisse aus der europäischen Debatte um nachhaltige Raumentwicklung widmet sich $\mathrm{Mi}$ chael Narodoslawsky aus Graz dem veränderten Wertekanon der Gesellschaft und der grundlegenden Neustrukturierung bei der Aushandlung raumwirksamer Problemstellungen. Erfahrungen mit Kommunikation für Nachhaltigkeit im Verwaltungshandeln und in der Zusammenarbeit mit Unternehmen thematisieren Petra
Heuer und Angela Schilde. Timothy Moss hinterfragt in seinem Beitrag den vorherrschenden positiven Blick auf regionale Partnerschaften und ihre Bedeutung für nachhaltige Entwicklung. Eckhard Bergmann behandelt die Chancen und Grenzen von Kooperation und Wettbewerb bzw. Konkurrenz im regionalen Kontext. Den Abschluss bildet der Beitrag von Frauke Burgdorff und Ralf Meyer, die über die ersten Erfahrungen und Anforderungen an ein Kommunikationsmanagement im Rahmen einer trinationalen Regionalentwicklung berichten.

\section{Anmerkungen}

(1) Vgl. z.B. Bundesamt für Bauwesen und Raumordnung(BBR) (Hrsg.): Handbuch Gute Beispiele einer nachhaltigen regionalen Raum- und Siedlungsentwicklung. Werkstatt: Praxis Nr. 1/2000, Bonn. www.zukunftsregionen.de. Das IÖW hat für das BBR 1998/99 den Wettbewerb Regionen der Zukunft wissenschaftlich begleitet. Dabei wurden bundesweit 422 lokal-regionale Projekte mit einem Fragebogen erfasst, von denen 60 als gute Beispiele für das Handbuch ausgewählt wurden. In einer neuen Studie (2001/2002) werden im Auftrag des BBR die damals erfassten Projekte mit Blick auf die Erfolgs- und Misserfolgsbedingungen einer Evaluierung unterzogen.

(2) Nischwitz, G./ Molitor, R./ Rohne, S.: Local und Regional Governance für eine nachhaltige Entwicklung? Schriftenreihe des IÖW, in Vorbereitung, Berlin. Siehe auch Nischwitz, G./ Molitor, R.: Regional Governance - Motor einer nachhaltigen Entwicklung? In: Ökologisches Wirtschaften, Nr. 3-4/2001, S. 24-25. Diese Beiträge sind Ergebnisse einer Sondierungsstudie im Rahmen des BMBF-Förderschwerpunkts Sozial-ökologische Forschung. Im Rahmen einer internationalen Expertenbefragung wurden Governance-Strukturen als eigenständiges Steverungsinstrumentarium behandelt und Erfahrungswissen über lokale und regionale Governance-Styles erfasst und abgeglichen. Dabei wurden Kernelemente von Regional Governance identifiziert und offene Forschungsfragen formuliert.

(3) Molitor, R. / Nischwitz, G. (Hrsg.): Kommunikation für eine nachhaltige Entwicklung in der Region - KOMREG.

Schriftenreihe des IÖW, in Vorbereitung, Berlin.

\section{Die Autoren}

Dr. Reimar Molitor ist bei der Regionale 2006 Agentur im Bergischen Städtedreieck für Regionalmanagement und Projektentwicklung zuständig. Dr. Guido Nischwitz leitet das Forschungsfeld Regionale Wirtschaftspolitik/ Nachhaltige Regionalentwicklung am Institut für ökologische Wirtschaftsforschung (IÖW) Kontakt: IÖW, Regionalbüro Nordrhein-Westfalen, Völklinger Str. 9, 42285 Wuppertal. Tel. 0202/ 80530, Fax 83402, E-Mail: mailbox@wuppertal.ioew.de 
(c) 20I0 Authors; licensee IÖW and oekom verlag. This is an article distributed under the terms of the Creative Commons Attribution Non-Commercial No Derivates License (http://creativecommons.org/licenses/by-nc-nd/3.o/), which permits unrestricted use, distribution, and reproduction in any medium, provided the original work is properly cited. 